\title{
Telomere shortening and DNA damage in culprit cells of different types of progressive fibrosing interstitial lung disease
}

\author{
Aernoud A. van Batenburg (10) ${ }^{1}$, Karin M. Kazemier ${ }^{2,3}$, \\ Matthijs F.M. van Oosterhout ${ }^{4}$, Joanne J. van der Vis ${ }^{1,5}$, Jan C. Grutters ${ }^{1,3}$, \\ Roel Goldschmeding ${ }^{6}$ and Coline H.M. van Moorsel ${ }^{1,3}$
}

Affiliations: ${ }^{1}$ Dept of Pulmonology, St Antonius ILD Center of Excellence, St Antonius Hospital, Nieuwegein, The Netherlands. ${ }^{2}$ Center of Translational Immunology, University Medical Center Utrecht, Utrecht, The Netherlands. ${ }^{3}$ Division of Heart and Lungs, University Medical Center Utrecht, Utrecht, The Netherlands. ${ }^{4}$ Dept of Pathology, Pathology DNA, St Antonius ILD Center of Excellence, St Antonius Hospital, Nieuwegein, The Netherlands. ${ }^{5}$ Dept of Clinical Chemistry, St Antonius ILD Center of Excellence, St Antonius Hospital, Nieuwegein, The Netherlands. ${ }^{6}$ Dept of Pathology, University Medical Center Utrecht, Utrecht, the Netherlands.

Correspondence: Coline H.M. van Moorsel, Interstitial Lung Diseases Center of Excellence, Dept of Pulmonology, St Antonius Hospital, Koekoekslaan 1, 3435 CM Nieuwegein, The Netherlands;

E-mail: c.van.moorseldantoniusziekenhuis.nl

ABSTRACT Pulmonary fibrosis is strongly associated with telomere shortening and increased DNA damage. Key cells in the pathogenesis involve alveolar type 2 (AT2) cells, club cells and myofibroblasts; however, to what extent these cells are affected by telomere shortening and DNA damage is not yet known. We sought to determine the degree of, and correlation between, telomere shortening and DNA damage in different cell types involved in the pathogenesis of progressive fibrosing interstitial lung disease. Telomere length and DNA damage were quantified, using combined fluorescence in situ hybridisation and immunofluorescence staining techniques, in AT2 cells, club cells and myofibroblasts of controls and patients with pulmonary fibrosis and a telomerase reverse transcriptase mutation (TERT-PF), idiopathic pulmonary fibrosis (IPF) and fibrotic hypersensitivity pneumonitis (fHP). In IPF and TERT-PF lungs, AT2 cells contained shorter telomeres and expressed higher DNA damage signals than club cells and myofibroblasts. In fHP lungs, club cells contained highly elevated levels of DNA damage, while telomeres were not obviously short. In vitro, we found significantly shorter telomeres and higher DNA damage levels only in AT2 surrogate cell lines treated with telomerase inhibitor BIBR1532. Our study demonstrated that in IPF and TERT-PF lungs, telomere shortening and accumulation of DNA damage primarily affects AT2 cells, further supporting the importance of AT2 cells in these diseases, while in fHP the particularly high telomere-independent DNA damage signals in club cells underscores its bronchiolocentric pathogenesis. These findings suggest that cell type-specific telomere shortening and DNA damage may help to discriminate between different drivers of fibrogenesis.

@ERSpublications

In patients with IPF, telomere shortening and accumulation of DNA damage primarily affects AT2 cells, while in fHP, the particularly high telomere-independent DNA damage signals in club cells underscore its bronchiolocentric pathogenesis https://bit.ly/35mP1JI

Cite this article as: van Batenburg AA, Kazemier KM, van Oosterhout MFM, et al. Telomere shortening and DNA damage in culprit cells of different types of progressive fibrosing interstitial lung disease. ERJ Open Res 2021; 7: 00691-2020 [https://doi.org/10.1183/23120541.00691-2020].

This article has supplementary material available from openres.ersjournals.com

Received: 23 Sept 2020 | Accepted: 14 Dec 2020

Copyright $\odot$ ERS 2021. This article is open access and distributed under the terms of the Creative Commons Attribution Non-Commercial Licence 4.0 


\section{Introduction}

Progressive fibrosing interstitial lung disease (ILD) is a group of devastating disorders characterised by scarring of the epithelium and reduced survival [1,2]. Although the pathogenesis is incompletely understood, evidence is growing that processes associated with accelerated ageing, such as telomere shortening and genetic instability, play a causative role in the destruction of the lung epithelium and subsequent fibrosis $[3,4]$.

Telomeres are DNA-protein complexes at the end of chromosomes which act as a buffer in cell cycle-dependent DNA shortening, thereby protecting the genetic information of the genome [5-7]. Shortening of telomeres is associated with several forms of progressive fibrosing ILD, such as idiopathic pulmonary fibrosis (IPF) and fibrotic hypersensitivity pneumonitis (fHP) [8-11]. In a subset of these patients, disease is caused by genetic mutations in telomere-related genes such as telomerase reverse transcriptase (TERT), the catalytic subunit of telomerase involved in telomere elongation and maintenance [12-16]. Furthermore, we have previously shown that telomere length in lung tissue of sporadic IPF patients is significantly reduced and associated with poorer survival $[8,17]$.

Critical telomere shortening is recognised as DNA damage similar to a double-strand DNA break. This results in the phosphorylation of $\mathrm{H} 2 \mathrm{~A}$ histone family member $\mathrm{X}(\gamma \mathrm{H} 2 \mathrm{AX})$ initiating the DNA damage response. In healthy circumstances, double-strand DNA breaks take approximately $72 \mathrm{~h}$ to resolve [18]. However, if not fixed properly in time, the DNA damage response becomes persistent and eventually leads to cellular senescence [19-21], a process associated with fibrogenesis in IPF lungs and fibrotic mouse models $[22,23]$. Previous studies using mice models with a telomere repeat factor-1 (Trf1) knockout in alveolar or bronchiolar epithelial cells demonstrated telomere shortening and increased DNA damage foci in both cell types $[24,25]$. Similar results were found in TRF2-inactivated human cell lines showing an elevated amount of damage foci at uncapped telomeres [26]. Furthermore, we previously found a significant inverse correlation between $\gamma \mathrm{H} 2 \mathrm{AX}$ signals and telomere length in alveolar epithelial cells of a patient with pulmonary fibrosis harbouring a PARN mutation [27]. In contrast, other studies reported that, even though DNA damage signals were increased, no such correlation between average whole lung telomere length and DNA damage signals was found in IPF lungs [28]. However, extensive cell type-specific measurements are missing in pulmonary fibrosis.

Several cell types have been associated with the pathogenesis of tissue remodelling underlying pulmonary fibrosis. Alveolar type 2 (AT2) cells, progenitor cells responsible for maintenance and renewal of the alveolar compartment, are generally considered to play a fundamental role in the onset of fibrogenesis in the lung $[24,29,30]$. Loss of functional AT2 cells results in an impaired renewal capacity of alveolar cells and the production of pro-fibrotic factors, subsequently leading to activation of myofibroblasts and extracellular matrix deposition [31]. However, there has been emerging interest in a role of club cells in pulmonary fibrosis. Similar to AT2 cells in alveoli, club cells are progenitor cells responsible for maintenance and renewal of bronchiolar epithelium. In pulmonary fibrosis, club cells drive bronchiolisation, a process in which bronchiolar epithelial cells migrate to and repopulate the alveoli [32, 33]. A third cell type, i.e. the myofibroblast, is the main source of collagen deposition, thereby driving fibrogenesis. Clusters of myofibroblasts forming fibroblast foci are a histological hallmark of IPF $[1,34]$. We previously found that in IPF lungs, telomeres in AT2 cells were shorter than in other, as yet unclassified cells surrounding these AT2 cells [8]. However, telomere length in the two other cell types involved in fibrogenesis has never been studied specifically in IPF or other types of pulmonary fibrosis such as fHP. Moreover, a possible quantitative and cell type-specific relationship between telomere shortening and persistent activation of the DNA damage response remains to be elucidated.

To mimic lung-specific telomere shortening in an experimental set-up, lung cell lines can be treated with BIBR1532, a telomerase inhibitor that binds noncompetitively to the active site of the telomerase protein [35]. Previously, BIBR1532-dependent telomere shortening in an A549 carcinoma cell line, which is derived from the alveolar epithelium and closely resembles AT2 cells, showed that after 140 population doublings telomeres were shorter in these cells [36]. However, to date telomere shortening in surrogate cell lines of club cells and myofibroblasts with BIBR1532 treatment and its correlation with DNA damage has not been assessed.

\section{Materials and methods}

\section{Patient selection}

Lung material from three patient groups with progressive fibrosing ILD were included in the analysis, consisting of 32 patients with IPF, 17 patients with pulmonary fibrosis and a TERT mutation (TERT-PF), and 9 patients with fHP (table S1). The study was approved by Medical Research Ethics Committees United, St Antonius Hospital (approval number W14.056 and R05-08A) and all patients provided written informed consent. 


\section{Cell culture}

To investigate the relationship between telomere shortening and DNA damage, nonsmall cell lung cancer cell lines A549 (cultured in Dulbecco's modified Eagle's medium (DMEM)) and NCI-H460 (cultured in Roswell Park Memorial Institute (RPMI) 1640 medium), bronchial epithelial cell line 16HBE (cultured in Minimum Essential Medium (MEM)) and lung fibroblast cell line MRC5 (cultured in Dulbecco's modified Eagle's medium/Nutrient Mixture F-12 (DMEM/F-12)) were treated with 0,10 or $25 \mu \mathrm{M}$ telomerase inhibitor 2-[(E)-3-naphthalen-2-yl-but-2-enoylamino]-benzoic acid (BIBR1532; Selleckchem, Munich, Germany). Additional detail on cell culture experiments is provided in the online data supplement.

\section{Whole biopsy telomere length measurements in lung tissue}

Whole biopsy telomere length in DNA extracted from formalin-fixed paraffin-embedded (FFPE) tissue was measured by monochrome multiplex quantitative polymerase chain reaction (MMqPCR) as described previously [8, 17, 37]. Thirteen controls, 17 TERT-PF, 32 IPF and 9 fHP lung biopsy samples were included. Additional detail on telomere length measurements by MMqPCR is provided in an online data supplement.

\section{Cell type-specific telomere and DNA damage staining}

Subsequent FFPE tissue slides of $4 \mu \mathrm{m}$ were prepared and stained for telomere length and DNA damage analysis in specifically labelled AT2 cells, club cells and myofibroblasts as described previously [27]. To investigate cell type-specific telomere length, we performed a fluorescence in situ hybridisation (FISH) analysis in a random sub-selection of control $(n=8)$, TERT-PF $(n=6), \operatorname{IPF}(n=10)$ and fHP $(n=5)$ lungs. Additional detail on telomere length and $\gamma \mathrm{H} 2 \mathrm{AX}$ DNA damage measurements by FISH and immunofluorescence (IF) is provided in an online data supplement.

\section{Statistical analysis}

Statistical significances were computed using nonparametric tests in GraphPad Prism version 8 (GraphPad Software, San Diego, CA, USA). Telomere length and DNA damage signal differences were determined by Mann-Whitney tests and combined Kruskal-Wallis and Dunn's multiple comparisons tests. First, we used the data to compare differences in the signal between the different groups of patients (controls, TERT-PF, IPF and fHP), then we used the data to compare differences between the different types of cells (AT2, club cells and myofibroblasts). Spearman's rank coefficient was used to calculate correlations per disease group between telomere length and $\gamma \mathrm{H} 2 \mathrm{AX}$ signal in all cell types together. Next, we investigated whether the correlation that was present in TERT-PF lungs would fit observed values in the specific cell types AT2, club cells or myofibroblasts in IPF or fHP lungs. The cell type-specific observed telomere length was used in the equation representing the correlation in TERT-PF lungs to calculate the cell type-specific expected DNA damage value. Statistical differences between observed and expected values for each cell type were computed using Mann-Whitney tests.

\section{Results}

No difference in whole biopsy telomere length between IPF and fHP lungs

Analysis of whole biopsy telomere length measured by MMqPCR showed that telomeres in the control group were significantly longer than in the patient groups $(\mathrm{p}<0.05)$. Furthermore, comparison between patient groups showed that telomere length in TERT-PF lungs was significantly shorter than in IPF and fHP groups $(\mathrm{p}<0.05)$. No difference was found between whole biopsy telomere length of IPF and fHP groups (table 1 ).

\section{AT2 cells of TERT-PF and IPF lungs have the shortest telomeres}

To determine cell type-specific telomere length, we stained telomeres by FISH together with cell type markers by IF (figure 1). Comparison between cell types showed that in controls there was no difference between telomere length in AT2 cells and club cells $(\mathrm{p}=0.961)$ (figure 2a). In TERT-PF and IPF lungs, telomere length in AT2 cells was significantly shorter than in club cells $(p<0.0001)$ (figure $2 b$ and $c)$ and in myofibroblasts $(\mathrm{p}<0.0001)$ (figure $2 \mathrm{~b}$ and $\mathrm{c}$ ), while in fHP lungs telomere length of AT2 cells was no different from that of club cells $(\mathrm{p}=0.168)$ (figure $2 \mathrm{~d}$ ), and slightly shorter than that of myofibroblasts $(\mathrm{p}=0.0002)$ (figure 2e). In all three patient groups, no difference in telomere length was found between club cells and myofibroblasts. Comparison between groups showed that telomere lengths in AT2 cells and club cells of control lungs were significantly longer than those of TERT-PF, IPF and fHP lungs (table 1). In contrast, telomere length in AT2 cells, club cells and myofibroblasts of TERT-PF lungs were significantly shorter than those of control, IPF and fHP lungs (table 1). Interestingly, telomeres in AT2 cells of IPF lungs were significantly shorter than in AT2 cells of fHP lungs $(p<0.0001)$, while telomere length in club cell and myofibroblasts did not differ between IPF and fHP (table 1). 


\section{TABLE 1 Group comparison of telomere length and DNA damage in lung tissue}

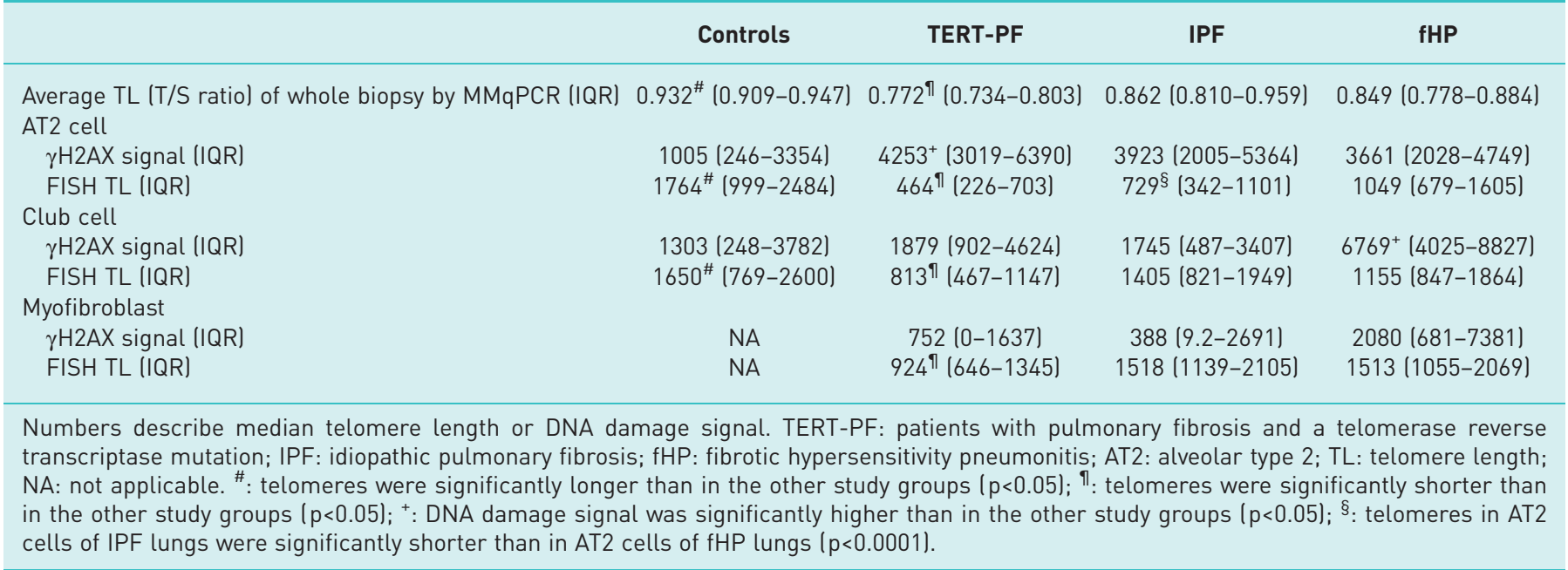

Increased DNA damage in club cells of fHP lungs

To determine cell type-specific DNA damage, we used immunofluorescent staining of $\gamma \mathrm{H} 2 \mathrm{AX}$ together with cell type-specific markers. Comparison between cell types showed that in TERT-PF and IPF lungs, the $\gamma \mathrm{H} 2 \mathrm{AX}$ signal in AT2 cells was significantly higher than in club cells $(\mathrm{p}<0.0001)$ (figure $2 \mathrm{f}$ and $\mathrm{g}$ ) and in myofibroblasts $(\mathrm{p}<0.0001)$ (figure $2 \mathrm{f}$ and $\mathrm{g}$ ). In contrast, in fHP lungs the $\gamma \mathrm{H} 2 \mathrm{AX}$ signal in club cells was higher than in AT2 cells $(\mathrm{p}<0.0001)$ (figure $2 \mathrm{~h}$ ) and myofibroblasts $(\mathrm{p}<0.0001)$ (figure $2 \mathrm{~h})$. Moreover, comparison between study groups showed that club cells in fHP lungs contained a significantly higher $\gamma H 2 A X$ signal than club cells in control $(\mathrm{p}<0.0001)$, TERT-PF $(\mathrm{p}<0.0001)$ and IPF lungs $(\mathrm{p}<0.0001)$ (table 1). In AT2 cells of TERT-PF lungs, the $\gamma \mathrm{H} 2 \mathrm{AX}$ signal was higher than in the other study groups (controls: $\mathrm{p}<0.0001$, IPF: $\mathrm{p}=0.038$ and fHP: $\mathrm{p}=0.005$ ) (table 1) while no difference in the $\gamma \mathrm{H} 2 \mathrm{AX}$ signal of AT2 cells between fHP and IPF lungs was detected $(\mathrm{p}=0.248)$ (table 1$)$.

\section{DNA damage in club cells and myofibroblasts of fibrotic lungs is higher than expected from telomere shortening alone}

Next, using data from all cell types, we analysed the association between FISH telomere length and the $\gamma \mathrm{H} 2 \mathrm{AX}$ signal and found in TERT-PF lungs a moderately strong correlation $(\mathrm{r}=-0.689)(\mathrm{p}=0.003$, figure 3a). The correlation between telomere length and DNA damage in TERT-PF lungs, in which a causative mutation in the TERT gene underlies telomere shortening and disease, shows that telomere shortening is probably the cause of the observed increase in DNA damage. However, using data from all cell types, such a correlation was not found in the IPF or fHP groups. Next, we investigated whether a correlation exists for specific cell types in IPF and fHP, rather than in cell types combined. Therefore, we used the equation representing the correlation between telomere length and DNA damage found in TERT-PF lungs to calculate the expected DNA damage for each cell type based on the observed telomere length for each cell type in IPF and fHP. The expected and observed DNA damage signals per cell type are presented in figures $3 \mathrm{~b}-\mathrm{g}$. In AT2 cells of IPF and fHP there was no difference between expected and observed DNA damage signals (figure $3 \mathrm{~b}$ and e). However, in club cells and myofibroblasts of IPF and fHP lungs, observed DNA damage signals were significantly higher than the expected values $(p<0.05)$ (figure $3 \mathrm{c}, \mathrm{d}$, f and $\mathrm{g}$ ).

\section{Induced telomere shortening and increased DNA damage in AT2 surrogate cell lines}

In order to experimentally study whether telomere shortening causes an increase in DNA damage, we added BIBR1532, a highly specific telomerase inhibitor, to cultures of surrogate lung cell lines A549 and NCI-H460 for AT2 cells, 16HBE for club cells and MRC5 for myofibroblasts. Inhibition of telomerase showed that only in AT2 surrogate cell lines A549 and NCI-H460 were telomeres shortened significantly with $25 \mu \mathrm{M}$ BIBR1532 $(\mathrm{p}<0.05)$ (figure $4 \mathrm{a}$ and $\mathrm{b})$ and that the level of DNA damage increased significantly compared with no BIBR1532 ( $\mathrm{p}=0.0001$ ) (figure $4 \mathrm{a}, \mathrm{b}$, e and $\mathrm{f}$ ). In 16HBE and MRC5 cells treated with $25 \mu \mathrm{M}$ BIBR1532, however, telomeres did not shorten (figure $4 \mathrm{c}$ and d), while DNA damage increased to very high levels when compared with no BIBR1532 ( $\mathrm{p}=0.0001)$ (figure $4 \mathrm{~g}$ and $\mathrm{h}$ ). 


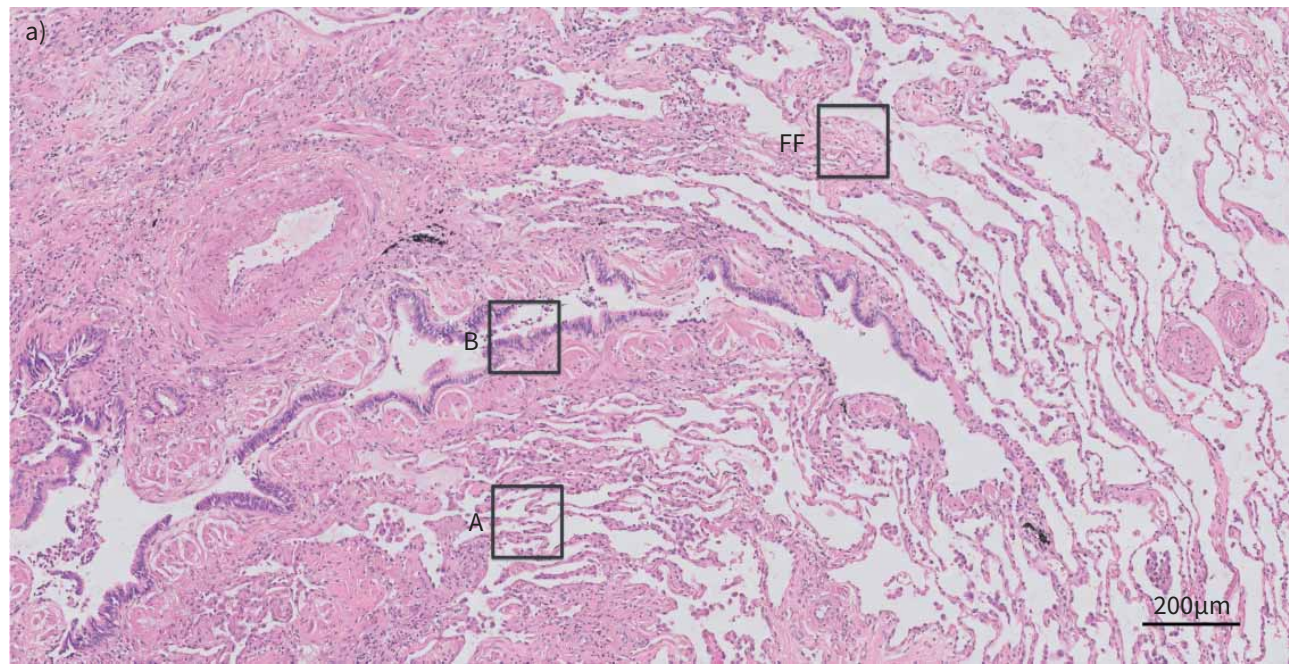

A: alveolar type 2

B: club cells

FF: myofibroblasts
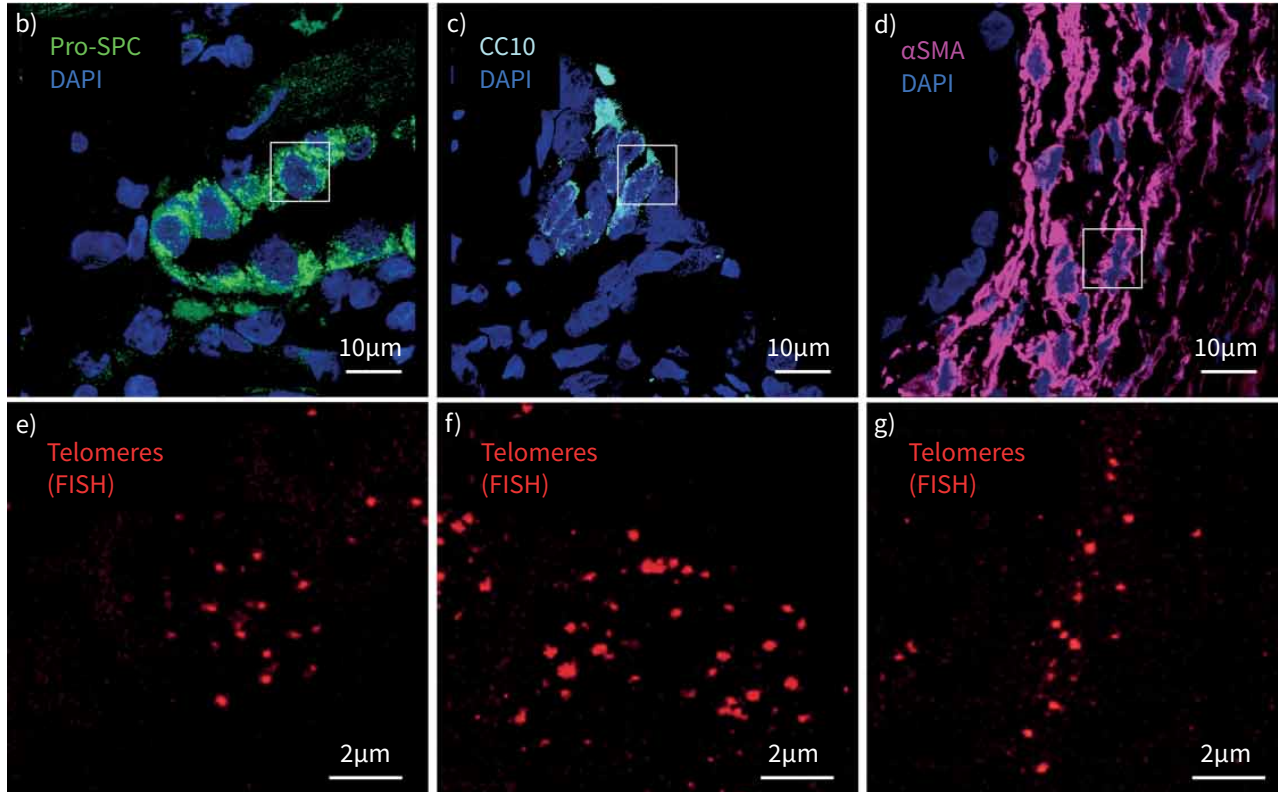

g)
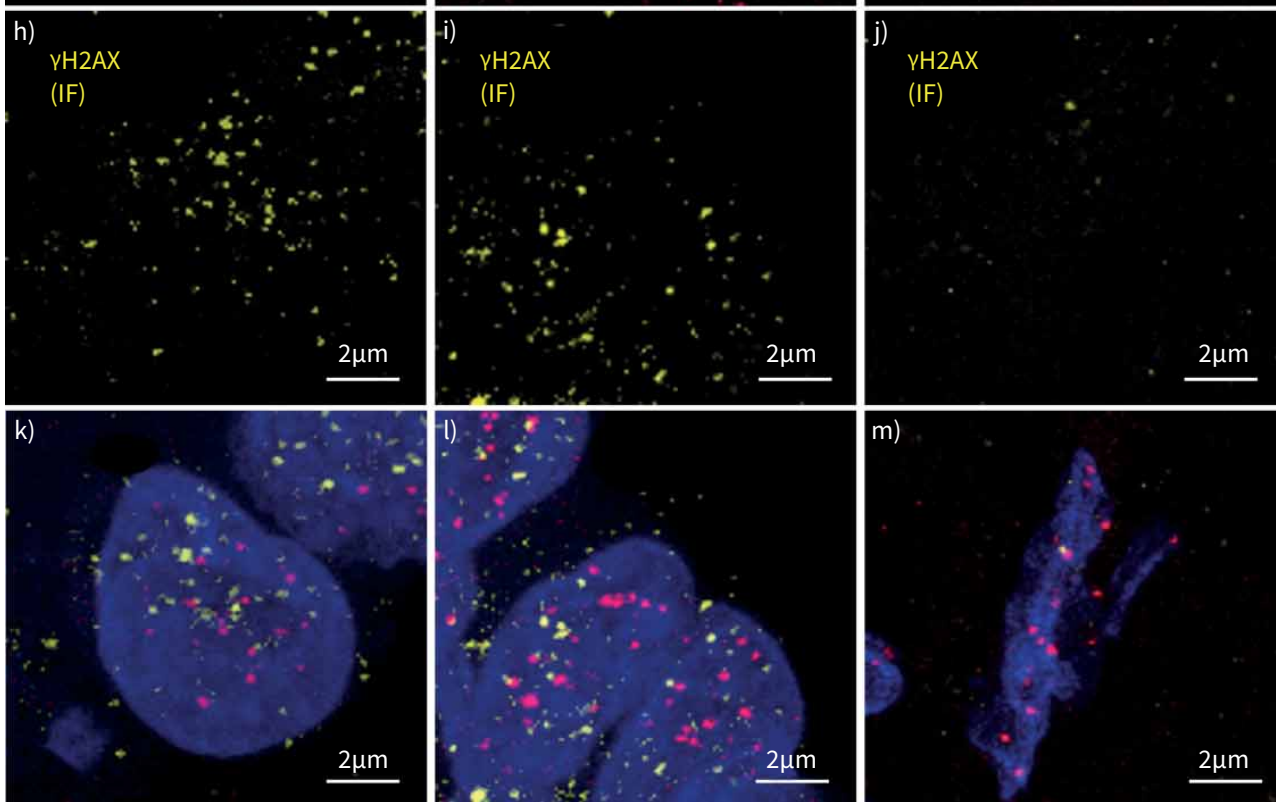
FIGURE 1 Telomere and DNA damage in fibrotic lung tissue measured by combined FISH and immunofluorescence staining techniques. a) Haematoxylin and eosin staining representing a typical idiopathic pulmonary fibrosis lung biopsy. b-d) Representative fluorescent stained examples of boxed areas in image (a), containing pro-surfactant protein C-positive alveolar type 2 cells (green) in the alveolus (A), CC10-positive club cells (light blue) in the bronchiolus (B) and $\alpha$-smooth muscle actin-positive myofibroblasts (purple), in the fibroblast focus (FF). e-g) FISH-stained telomere signals (red dots) and h-j) immunofluorescence-stained $\gamma \mathrm{H} 2 \mathrm{AX}$ signals (yellow dots) in magnified boxed areas of images (b-d). k-m) Overlay pictures of telomere, $\gamma \mathrm{H} 2 \mathrm{AX}$ and DAPI stainings. All fluorescent pictures were captured and Z-stacked using a LSM700 laser scanning confocal microscope. FISH: fluorescence in situ hybridisation; DAPI: 4',6-diamidino-2-phenylindole; $\gamma \mathrm{H} 2 \mathrm{AX}$ : phosphorylated histone protein from the H2A family.

\section{Discussion}

In this study, telomere length and DNA damage were investigated for the first time in different cell types involved in the pathogenesis of progressive fibrosing ILD. In AT2 cells of patients with IPF and patients with a TERT mutation we detected the shortest telomeres and highest DNA damage signals when compared to club cells and myofibroblasts. However, telomere length in AT2 cells of fHP lungs was not obviously short, while very high DNA damage signals were present in club cells. The observed increase of DNA damage in AT2 cells may be caused by telomere shortening. This was experimentally replicated in two AT2 surrogate cell lines, which showed BIBR1532-induced telomere shortening together with increased DNA damage. However, the level of DNA damage in club cells and myofibroblasts of IPF and fHP lungs could not be explained by telomere shortening alone.

a)

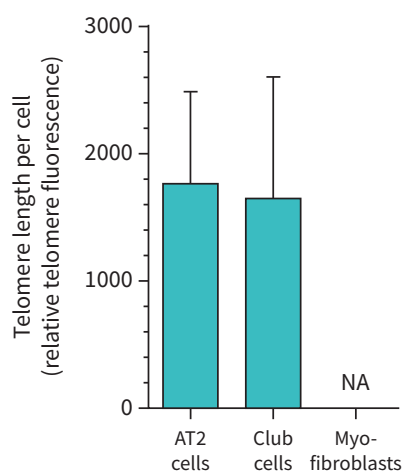

e)

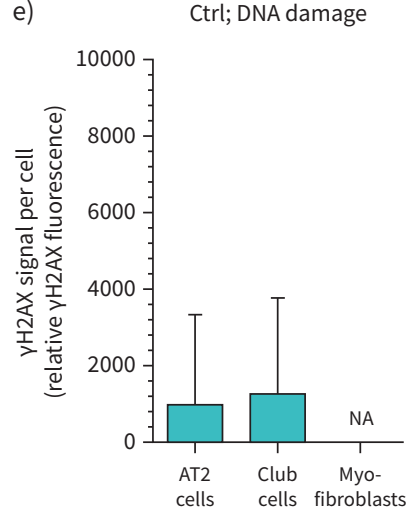

b)

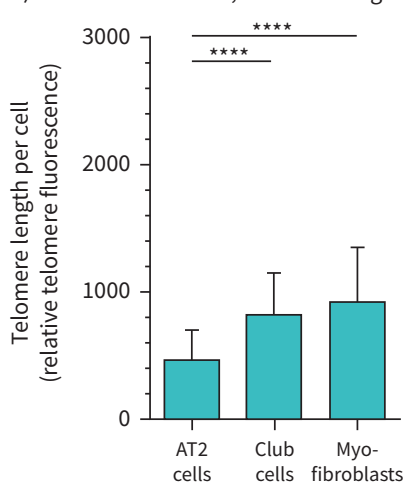

f)

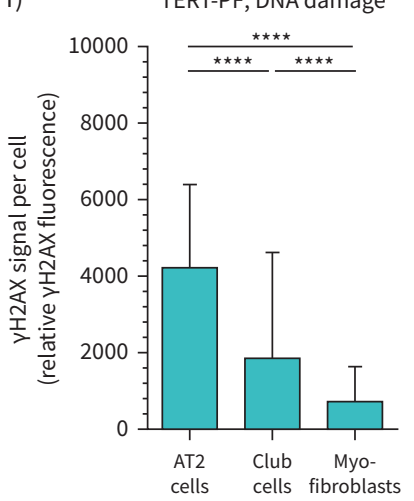

c)

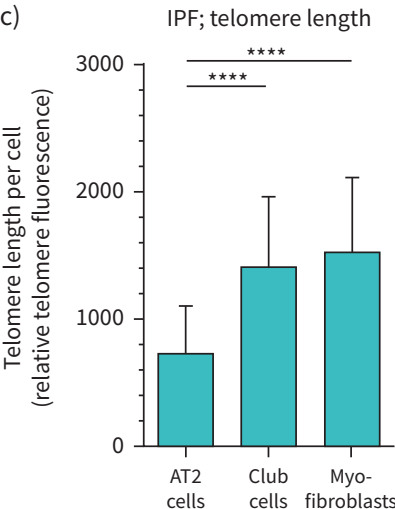

g)

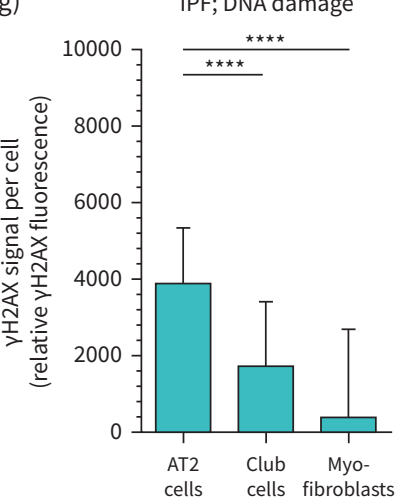

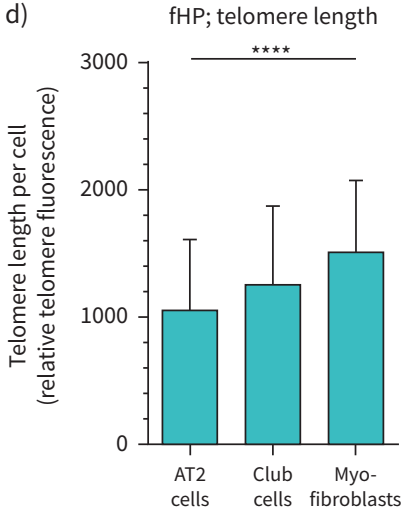

h)

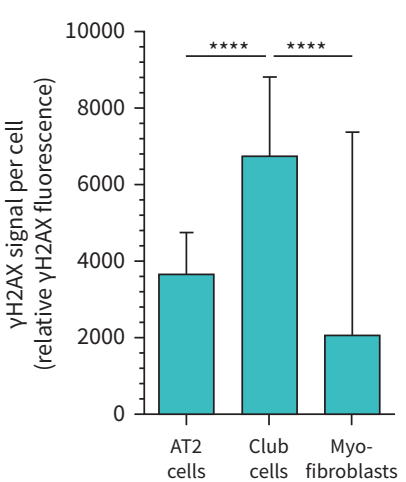

FIGURE 2 Cell-specific quantification of telomere and DNA damage signals in control and fibrotic lungs. Bar charts of telomere length (measured by FISH) and DNA damage signals (measured by immunofluorescence) in alveolar type 2 (AT2) cells, club cells and myofibroblasts in lungs of $a$ and e) eight controls, $b$ and f) six patients with pulmonary fibrosis and a telomerase reverse transcriptase (TERT-PF) mutation, $c$ and g) 10 idiopathic pulmonary fibrosis (IPF) and $d$ and h) five fibrotic hypersensitivity pneumonitis (fHP) patients. Controls contained long telomeres and low DNA damage signals in AT2 cells and club cells. In TERT-PF and IPF lungs, telomere length in AT2 cells was shorter than in club cells and myofibroblasts ( $p<0.0001)$ and DNA damage signals in AT2 cells were significantly higher than in club cells and myofibroblasts ( $p<0.0001)$. In fHP lungs, DNA damage signals in club cells were highly elevated compared to AT2 cells and myofibroblasts $(p<0.0001)$, while telomere length in club cells was comparable with AT2 cells and myofibroblasts. Boxes represent medians and whiskers extend up to values within the third quartile. FISH: fluorescence in situ hybridisation; $\gamma \mathrm{H} 2 \mathrm{AX}$ : phosphorylated histone protein from the H2A family; Ctrl: control; NA: not applicable. ${ }^{* * * *}: \mathrm{p}<0.0001$, calculated by Kruskal-Wallis multiple comparison tests. 
a)

TERT-PF; AT2 cells, club cells and myofibroblasts
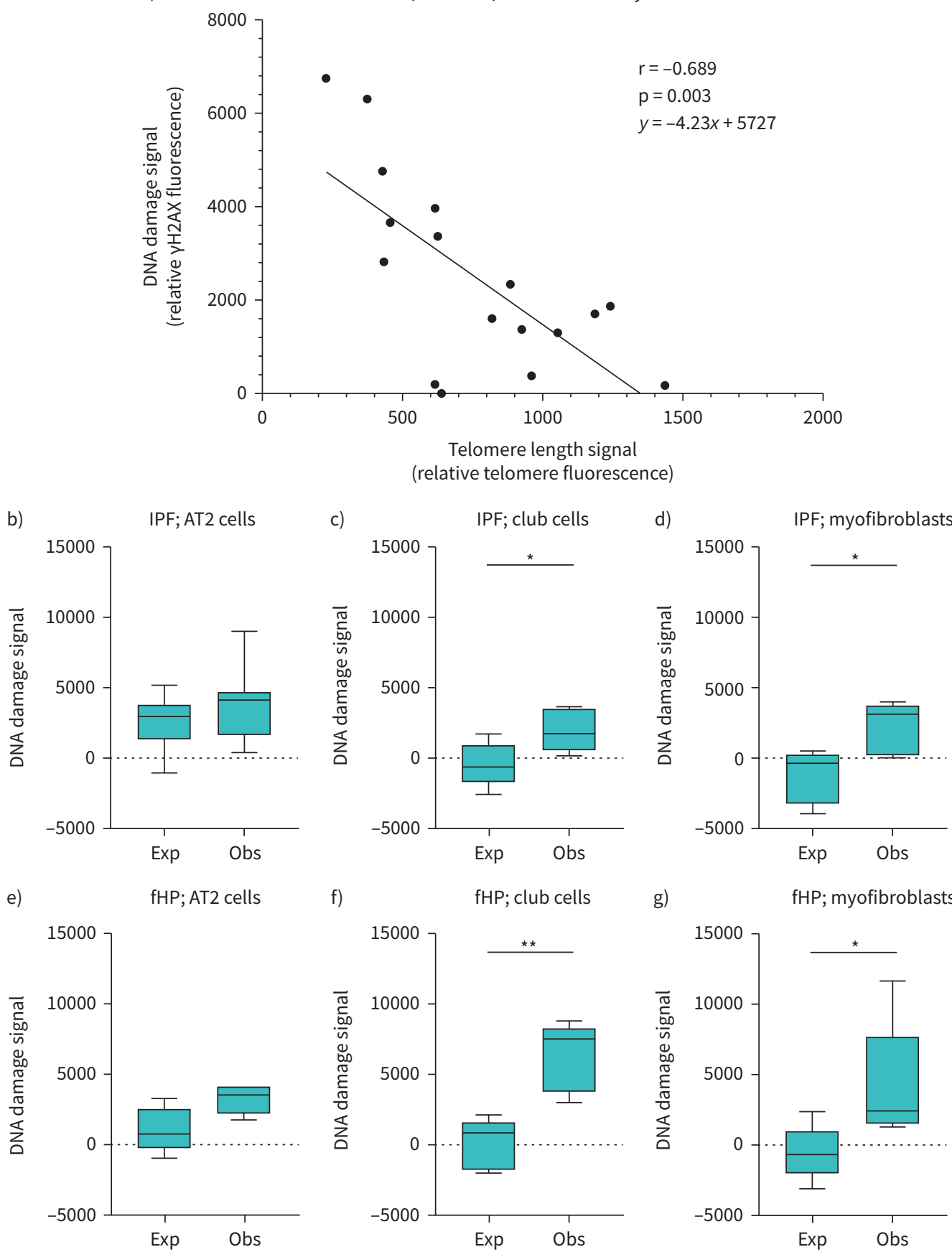

FIGURE 3 Observed (obs) and expected (exp) DNA damage signals in alveolar type 2 (AT2) cells, club cells and myofibroblasts of idiopathic pulmonary fibrosis (IPF) and fibrotic hypersensitivity pneumonitis (fHP) lungs. a) Inverse spearman correlation ( $r=-0.689, p=0.003)$ of median telomere length and median $\gamma \mathrm{H} 2 \mathrm{AX}$ signals in AT2 cells, club cells and myofibroblasts per biopsy specimen in six TERT-PF lungs resulting in a linear regression line with equation $y=-4.23 x+5727$. This equation was used to calculate expected DNA damage values from observed telomere length signals in b) IPF AT2 cells, c) IPF club cells, d) IPF myofibroblasts, e) fHP AT2 cells, f) fHP club cells, and g) fHP myofibroblasts. In IPF and fHP, club cells and myofibroblasts showed lower expected than observed DNA damage signals, while in AT2 cells no significant difference was found between expected and observed DNA damage signals. Statistical differences were computed by Mann-Whitney tests. $\gamma \mathrm{H} 2 \mathrm{AX}$ : phosphorylated histone protein from the H2A family; TERT-PF: patients with pulmonary fibrsosis and a telomerase reverse transcriptase (TERT) mutation. *: $p<0.05$; ${ }^{* *}: p<0.01$.

Cell type-specific analysis is most important to better understand processes in fibrotic lungs. Our results demonstrate that although no differences in average whole lung biopsy telomere length were present between IPF and fHP, significant differences between cell types exist. In both TERT-PF and IPF lungs, 
a)

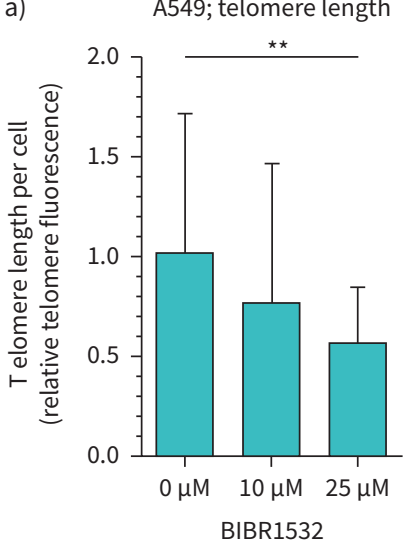

e)

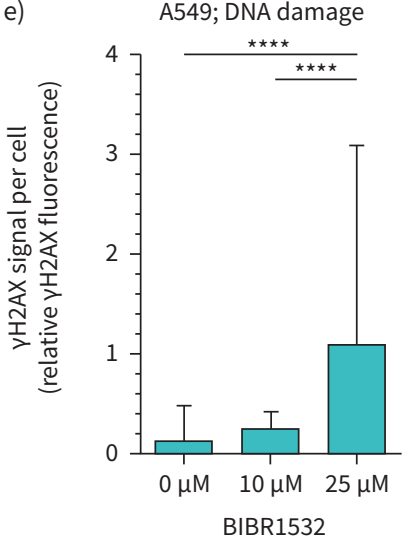

b)

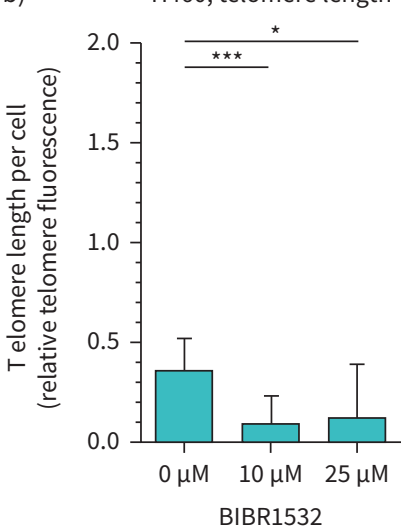

f)

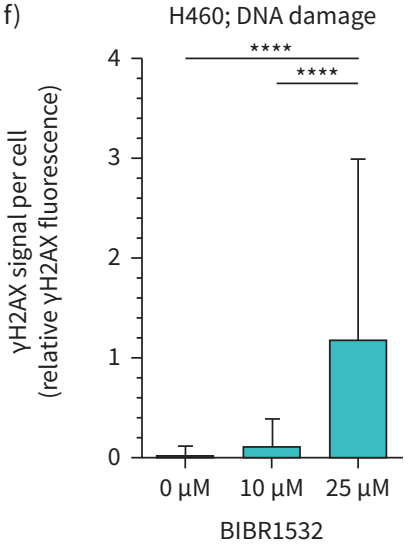

c)

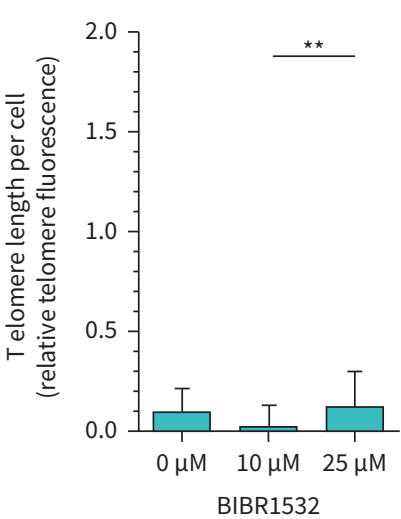

g)

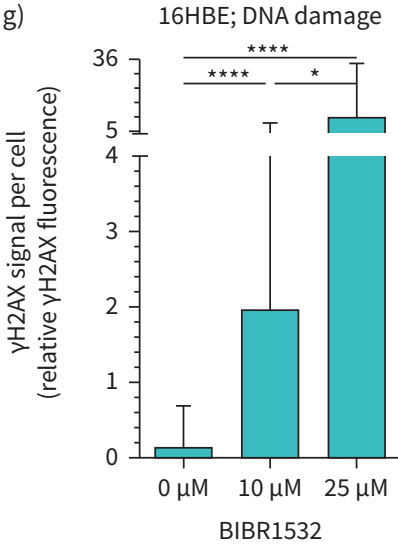

d)

MRC5; telomere length

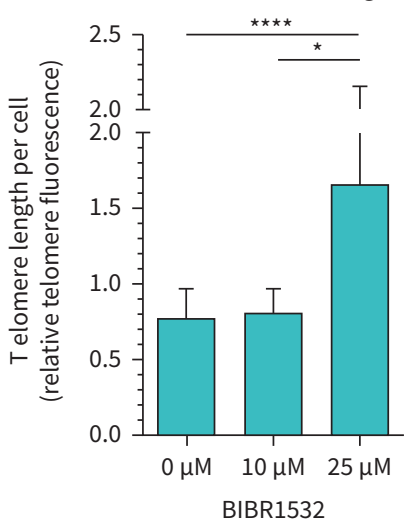

h)

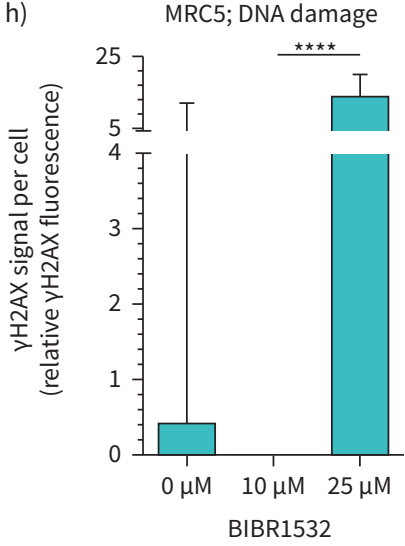

FIGURE 4 Experimental induction of telomere shortening and DNA damage signals by inhibition of telomerase by BIBR1532. Bar charts of telomere length (measured by FISH) and DNA damage signals (measured by immunofluorescence) in cytospins of a and e) A549, b and f) H460, $c$ and g) $16 \mathrm{HBE}$ and $\mathrm{d}$ and h) MRC5 cell lines. Induced telomere shortening with $25 \mu \mathrm{M}$ BIBR1532 was only observed in A549 ( $p=0.004$ ) and H460 cells ( $p=0.013$ ) compared to untreated cells, while in all cell lines increased DNA damage was found. Telomeres in MRC5 cells treated with $25 \mu \mathrm{M}$ BIBR1532 were significantly longer than untreated cells ( $p=0.0001)$. Boxes represent medians and whiskers extend up to values within the third quartile. FISH: fluorescence in situ hybridisation; $\gamma \mathrm{H} 2 \mathrm{AX}$ : phosphorylated histone protein from the H2A family; BIBR1532: 2-[(E)-3-naphthalen-2-yl-but-2-enoylamino]-benzoic acid. ${ }^{*}: p<0.05 ;{ }^{* *}: p<0.01 ;{ }^{* * *}: p<0.001 ;{ }^{* * * *}$ : $p<0.0001$, calculated by Kruskal-Wallis multiple comparison tests.

telomere length in AT2 cells was most affected, confirming the important role of AT2 cell telomere shortening in IPF pathogenesis $[8,10,24,30]$. Moreover, $\gamma \mathrm{H} 2 \mathrm{AX}$ signals were significantly elevated in AT2 cells of these groups. However, only in TERT-PF lungs, in which a causative mutation in the TERT gene underlies telomere shortening and disease, was there a correlation between telomeres and $\gamma \mathrm{H} 2 \mathrm{AX}$-related DNA damage, suggesting that telomere shortening causes DNA damage in these patients. This is consistent with a previous report showing that in IPF lungs no correlation was found between average whole lung telomere length and DNA damage signals [28] and supporting the notion that DNA damage in IPF may be caused by other factors than telomere shortening. To investigate whether a decrease in telomere length induces an increase in DNA damage for each cell type in IPF and fHP lungs, we tested whether observed DNA damage signals deviated from expected DNA damage signals based on the observed telomere length. In AT2 cells, observed and expected DNA damage signals were similar, while in club cells and myofibroblasts the amount of DNA damage was significantly higher than expected. These data imply that in AT2 cells of IPF and fHP lungs, telomere shortening may be the primary cause of DNA damage, while in club cells and myofibroblasts other processes may be involved.

Progressive telomere shortening and accumulation of DNA damage are prominent features of ageing and may eventually lead to cellular senescence or apoptosis [19-21]. Our data demonstrate that telomere shortening in TERT-PF lungs and in AT2 cells of IPF and fHP lungs is associated with elevated DNA damage signals. This suggests that AT2 cells in fibrotic lungs are prone to becoming senescent or apoptotic. Senescent and apoptotic AT2 cells have been observed in IPF [31, 38, 39] but not in fHP lungs. Accumulation of senescent cells has been associated with progressive pulmonary fibrosis in IPF lungs and fibrotic mouse models [22, 23, 40,41] and was postulated to drive pulmonary dysfunction in IPF. 


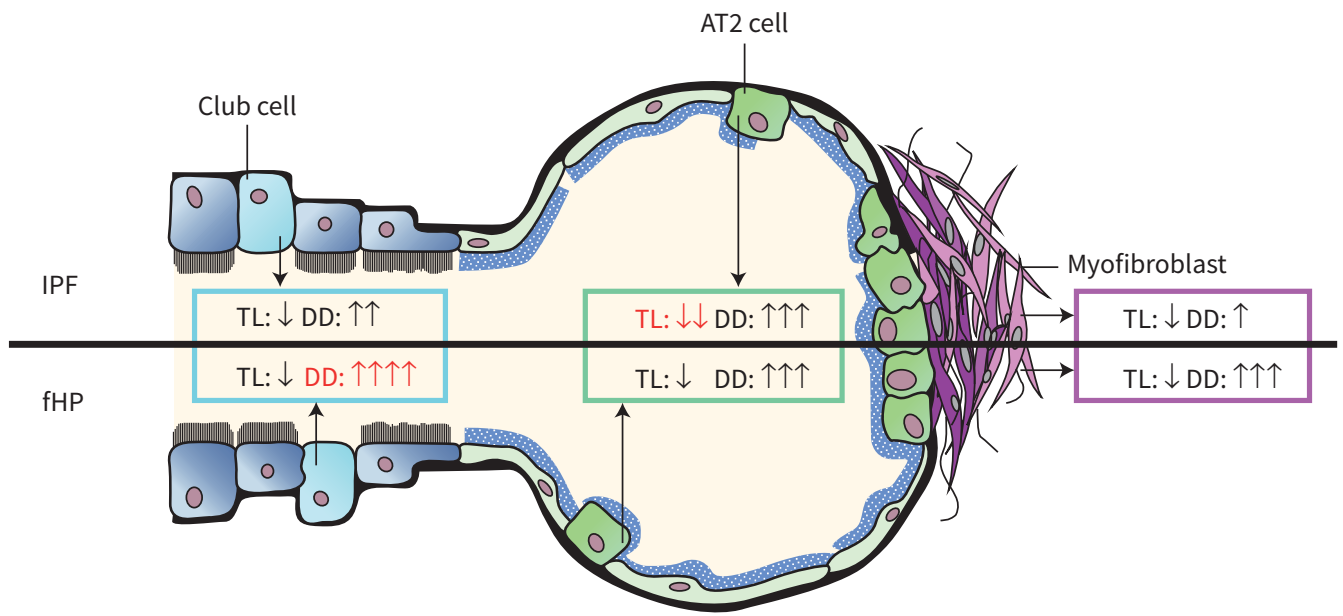

DNA damage (DD) is highest in club cells of fHP lungs

FIGURE 5 Graphical illustration summarising the main message of this study. The extent to which key cells in progressive fibrosing lung disease are affected by telomere shortening and DNA damage is so far unknown. This study revealed that in patients with idiopathic pulmonary fibrosis (IPF), telomere shortening and accumulation of DNA damage is primarily affecting alveolar type 2 (AT2) cells, further supporting the importance of AT2 cells in this disease, while in fibrotic hypersensitivity pneumonitis (fHP) the particularly high telomere-independent DNA damage signals in club cells underscores its bronchiolocentric pathogenesis.

Whether the high level of DNA damage observed in club cells of fHP lungs is associated with senescence or apoptosis remains to be investigated. This is of special interest because treatment with senolytic dasatinib in combination with quercetin was proven to be effective in eliminating cultured senescent human lung cells [42], and recent clinical trials with these drugs demonstrated physical alleviation in patients with IPF [43].

In fHP, club cells most prominently contained highly elevated DNA damage signals, but showed no excessive telomere shortening. The causal trigger of $\mathrm{fHP}$ is a sustained allergic reaction against an extrinsic antigen, but how this allergic reaction leads to telomere-unrelated DNA damage in club cells is unclear. A possible cause may be the inflammation-induced accumulation of reactive oxygen species (ROS), a group of highly reactive, DNA damage-inducing molecules that are also associated with other allergic diseases, such as asthma $[44,45]$. Because fHP is characterised by inhaled antigens that, due to size, strand in the bronchioles [46], it is possible that the accumulation of DNA damage in club cells of fHP lungs at this location is caused by ROS, and not by telomere shortening. This is in line with a previous study that showed that bronchoalveolar lavage of fHP patients contained significantly higher carbonylated protein levels, a marker of ROS, compared to IPF and controls [47]. However, another study showed that in mice with a club cell-specific knockout of telomere repeat-binding factor-1 (Trf1), rapid ageing of club cells by telomere dysfunction alone was sufficient to induce DNA damage and subsequent bronchiolocentric fibrosis [25]. In addition, it was reported that in $25 \%$ of the cases with an initial diagnosis of IPF, bronchiolocentric fibrosis is indicative for a revised diagnosis of fHP [48]. The excess DNA damage in fHP club cells might therefore, regardless of the cause, be suggestive of an important role for these cells in disease development and is in congruence with the localisation of fibrosis in fHP.

Next, we showed for the first time that experimental inhibition of telomerase resulted in telomere shortening in AT2 cell surrogate cell lines A549 and NCI-H460, suggesting that telomeres in these cells, similar to AT2 cells in diagnostic biopsies of pulmonary fibrosis, are most sensitive to telomerase dysfunction. This is in congruence with previous experiments where telomere shortening in BIBR1532-treated NCI-H460 cells was observed [36]. Moreover, in both in situ and in vitro experiments, a decrease in telomere length in AT2 cells is associated with an increase in DNA damage, underlining the telomere-dependent accumulation of DNA damage in AT2 cells. Furthermore, in surrogate club cell and myofibroblast cell lines 16HBE and MRC5 treated with BIBR1532, no evident telomere shortening was found, while these cells showed high levels of DNA damage. This corresponds with the finding that club cells and myofibroblasts in IPF and fHP tissue accumulate DNA damage independent of telomere shortening. However, it is unclear why BIBR1532-treated 16HBE and MRC5 cells present with high telomere-independent levels of DNA damage. 
Strengths of this study include the detailed assessment of cell type-specific telomere length and DNA damage in a broad spectrum of progressive fibrosing ILD patients, including those harbouring a TERT mutation (TERT-PF), IPF and fHP. However, some limitations are worth noting. The data presented here are based on associations; no causative links could be concluded from telomere length and DNA damage analysis in human tissue samples. Also, control tissue was obtained from various sources, such as residual lung resected from tissue next to a tumour. Furthermore, in contrast to the other cell lines, MRC5 cells are mortal foetal cells. Even though the MRC5 cells used in this study were still actively replicating, no definitive conclusions can be drawn on telomere length or DNA damage signals compared to other cell lines. Finally, use of primary cells instead of surrogate cell lines for AT2 and club cells would have been optimal to investigate a relationship between telomere shortening and DNA damage.

In conclusion, this is the first study to address in detail telomere status and DNA damage signals in AT2 cells, club cells and myofibroblasts in different types of progressive fibrosing ILD. In IPF and TERT-PF lungs, telomere shortening and accumulation of DNA damage primarily affects AT2 cells, further supporting their central role in fibrogenesis of these groups, while the remarkably high DNA damage in club cells of fHP lungs underscores the more bronchiolocentric fibrogenesis and a prominent role for club cells and DNA damage in fHP (figure 5). To further elucidate the link between club cells and the pathogenesis of fHP, future studies should focus on cellular ageing due to a sustained allergic reaction and DNA damage in these cells.

Conflict of interest: A.A. van Batenburg has nothing to disclose. K.M. Kazemier has nothing to disclose. M.F.M. van Oosterhout reports personal fees from Roche outside the submitted work. J.J. van der Vis has nothing to disclose J.C. Grutters has nothing to disclose. R. Goldschmeding has nothing to disclose. C.H.M. van Moorsel has nothing to disclose.

Support statement: This study was supported by the St Antonius Research Fund and ZonMW TopZorg St Antonius Science Corner grant 842002003. Funding information for this article has been deposited with the Crossref Funder Registry.

\section{References}

1 Raghu G, Collard HR, Egan JJ, et al. An official ATS/ERS/JRS/ALAT statement: idiopathic pulmonary fibrosis: evidence-based guidelines for diagnosis and management. Am J Respir Crit Care Med 2011; 183: 788-824.

2 Travis WD, Costabel U, Hansell DM, et al. An official American Thoracic Society/European Respiratory Society statement: update of the international multidisciplinary classification of the idiopathic interstitial pneumonias. $A m$ J Respir Crit Care Med 2013; 188: 733-748.

3 Martinez FJ, Collard HR, Pardo A, et al. Idiopathic pulmonary fibrosis. Nat Rev Dis Primers 2017; 3: 17074.

4 Selman M, Lopez-Otin C, Pardo A. Age-driven developmental drift in the pathogenesis of idiopathic pulmonary fibrosis. Eur Respir J 2016; 48: 538-552.

5 Chan SR, Blackburn EH. Telomeres and telomerase. Philos Trans R Soc Lond B Biol Sci 2004; 359: 109-121.

6 Maubaret CG, Salpea KD, Romanoski CE, et al. Association of TERC and OBFC1 haplotypes with mean leukocyte telomere length and risk for coronary heart disease. PLoS One 2013; 8: e83122.

7 Wyatt HD, West SC, Beattie TL. InTERTpreting telomerase structure and function. Nucleic Acids Res 2010; 38: 5609-5622.

8 Snetselaar R, van Batenburg AA, van Oosterhout MFM, et al. Short telomere length in IPF lung associates with fibrotic lesions and predicts survival. PLoS One 2017; 12: e0189467.

9 Everaerts S, Lammertyn EJ, Martens DS, et al. The aging lung: tissue telomere shortening in health and disease Respir Res 2018; 19: 95.

10 Alder JK, Chen JJ, Lancaster L, et al. Short telomeres are a risk factor for idiopathic pulmonary fibrosis. Proc Natl Acad Sci U S A 2008; 105: 13051-13056.

11 Snetselaar R, van Moorsel CH, Kazemier KM, et al. Telomere length in interstitial lung diseases. Chest 2015; 148 1011-1018.

12 Tsakiri KD, Cronkhite JT, Kuan PJ, et al. Adult-onset pulmonary fibrosis caused by mutations in telomerase. Proc Natl Acad Sci U S A 2007; 104: 7552-7557.

13 Alder JK, Cogan JD, Brown AF, et al. Ancestral mutation in telomerase causes defects in repeat addition processivity and manifests as familial pulmonary fibrosis. PLoS Genet 2011; 7: e1001352.

14 Armanios MY, Chen JJ, Cogan JD, et al. Telomerase mutations in families with idiopathic pulmonary fibrosis. N Engl J Med 2007; 356: 1317-1326.

15 Nakamura TM, Morin GB, Chapman KB, et al. Telomerase catalytic subunit homologs from fission yeast and human. Science 1997; 277: 955-959.

16 Ley B, Torgerson DG, Oldham JM, et al. Rare protein-altering telomere-related gene variants in patients with chronic hypersensitivity pneumonitis. Am J Respir Crit Care Med 2019; 200: 1154-1163.

17 van Batenburg AA, Kazemier KM, van Oosterhout MFM, et al. From organ to cell: multi-level telomere length assessment in patients with idiopathic pulmonary fibrosis. PLoS One 2020; 15: e0226785.

18 Ambrosio S, Di Palo G, Napolitano G, et al. Cell cycle-dependent resolution of DNA double-strand breaks. Oncotarget 2016; 7: 4949-4960.

19 Smogorzewska A, de Lange T. Different telomere damage signaling pathways in human and mouse cells. EMBO J 2002; 21: 4338-4348.

20 Chilosi M, Poletti V, Rossi A. The pathogenesis of COPD and IPF: distinct horns of the same devil? Respir Res 2012; 13: 3 . 
espir Crit Care Med 2021; 203: 707-717. pulmonary fibrosis. Am J Respir Crit Care Med 2012; 186: 306-313.

41 Hashimoto M, Asai A, Kawagishi H, et al. Elimination of p19(ARF)-expressing cells enhances pulmonary function in mice. JCI Insight 2016; 1: e87732.

42 Schafer MJ, White TA, Iijima K, et al. Cellular senescence mediates fibrotic pulmonary disease. Nat Commun 2017; 8: 14532

43 Justice JN, Nambiar AM, Tchkonia T, et al. Senolytics in idiopathic pulmonary fibrosis: results from a first-in-human, open-label, pilot study. EBioMedicine 2019; 40: 554-563.

44 Lonkar P, Dedon PC. Reactive species and DNA damage in chronic inflammation: reconciling chemical mechanisms and biological fates. Int J Cancer 2011; 128: 1999-2009.

45 Bowler RP, Crapo JD. Oxidative stress in allergic respiratory diseases. J Allergy Clin Immunol 2002; 110: 349-356.

46 Selman M, Pardo A, King TE Jr. Hypersensitivity pneumonitis: insights in diagnosis and pathobiology. Am J Respir Crit Care Med 2012; 186: 314-324.

47 Bargagli E, Penza F, Vagaggini C, et al. Analysis of carbonylated proteins in bronchoalveolar lavage of patients with diffuse lung diseases. Lung 2007; 185: 139-144.

48 Tanizawa K, Ley B, Vittinghoff E, et al. Significance of bronchiolocentric fibrosis in patients with histopathological usual interstitial pneumonia. Histopathology 2019; 74: 1088-1097. 